\title{
Morfofisiologia dos exercícios orofaríngeos: revisão integrativa
}

\author{
Oropharyngeal exercises morphophisiology: \\ an integrative review
}

\section{Morfofisiología de los ejercicios orofaríngeos: revisión integrativa}

\author{
Andresa Santos Silva* \\ Esther Mandelbaum Gonçalves Bianchini* \\ Ruth Ramalho Ruivo Palladino*
}

\section{Resumo}

Introdução: Dentre as possíveis abordagens terapêuticas fonoaudiológicas para as regiões orofacial e orofaríngea, a utilização de exercícios constitui-se em proposta frequente para trazer mudanças na musculatura envolvida e na funcionalidade dessas regiões. Entretanto, pouco se busca em relação à compreensão de como os exercícios funcionam para essa musculatura e principalmente quais as possíveis mudanças que podem ser observadas a partir de um programa de treinamento com exercícios. Objetivo: Realizar revisão integrativa da literatura científica referente à morfofisiologia dos exercícios orofaríngeos empregados na terapêutica fonoaudiológica. Método: Realizada busca nas bases de dados eletrônicas: Pubmed, Medline e Scielo, pela combinação dos seguintes Descritores em Ciências da Saúde (DeCs): "fisiologia", "exercício"; "terapia miofuncional"; "disfagia" e "Fonoaudiologia" nas línguas portuguesa e inglesa, por descritores associados (e/and). A seleção dos estudos foi realizada por meio da leitura do título, resumo, para aplicação dos critérios de inclusão e exclusão. Resultados: No total, foram encontrados 890 estudos nas bases de dados. A partir dos critérios de inclusão e exclusão foram selecionados 12 artigos, os quais foram analisados criteriosamente. Os artigos selecionados apresentaram quais músculos são ativados durante a prática dos exercícios, qual efetividade individual do exercício proposto e os casos em que essas terapêuticas são indicadas. Embora esses estudos tenham contribuído para a base de conhecimento atual, os mesmos possuem diferentes desenhos metodológicos. Conclusão: Parece existir reduzido número de estudos que abordem os efeitos promovidos pela terapia miofuncional orofacial nos músculos e funções orofaríngeas, sendo a maioria deles relacionados aos estudos da disfagia orofaríngea.

Palavras-chave: Fisiologia; Exercício; Orofaringe; Terapia Miofuncional; Fonoaudiologia.

\footnotetext{
* Pontifícia Universidade Católica de São Paulo- PUC-SP, São Paulo, SP, Brasil. Contribuição dos autores:

ASS: concepção do estudo, coleta de dados, redação do artigo; EMGB: método, revisão crítica e orientação do desenvolvimento da pesquisa; RRRP: revisão do manuscrito e orientação do desenvolvimento da pesquisa.
}

E-mail para correspondência: Andresa Santos Silva andresasantos.g3@gmail.com Recebido: $19 / 12 / 2018$

Aceito: 20/05/2019 
Abstract

Introduction: Among the possible speech-language therapy approaches for the orofacial and oropharyngeal regions, the use of exercises constitutes a frequent proposal to bring about changes in the musculature involved and the functionality of these regions. However, little is sought in relation to the understanding of how the exercises work for this musculature and especially what the possible changes that can be observed from the exercise training program. Objective: To carry out an integrative review of the scientific literature referring to the morphophysiology of the exercises oropharyngeal used speech therapy. Method: the bibliographic survey was carried out in the electronic databases: Pubmed, MEDLINE and Scielo, using the Descriptors in Health Sciences (DeCs): "exercise"; "physiology"; "myofunctional therapy"; "dysphagia" and "Speech, Language and Hearing Sciences" in Portuguese and English. Inclusion and exclusion criteria were applied based on the title and the abstracts. Results: In total, 890 studies were found in the databases. Based on the inclusion and exclusion criteria, 12 articles were analyzed carefully. The selected articles presented which muscles are activated during exercise, which individual effectiveness of the proposed exercise and the cases in which these therapies are indicated. Although these studies have contributed to the current knowledge base, they have different methodological designs. Conclusion: There seems to be a small number of studies addressing the effects promoted by orofacial myofunctional therapy in muscles and oropharyngeal functions. Most of them are related to the studies of oropharyngeal dysphagia.

Keywords: Physiology; Exercise; Oropharynx; Myofunctional Therapy; Speech therapy.

\section{Resumen}

Introducción: Entre los posibles abordajes terapéuticos fonoaudiológicos para regiones orofacial y orofaríngea, la utilización de ejercicios se constituye en propuesta frecuente para traer cambios en la musculatura involucrada y en la funcionalidad de esas regiones. Poco se busca en relación con la comprensión de cómo los ejercicios funcionan para esa musculatura y principalmente cuáles son los posibles cambios que pueden ser observados a partir de un programa de entrenamiento con ejercicios. Objetivo: Realizar revisión integrativa de la literatura científica referente a la morfofisiología de los ejercicios orofaríngeos empleados en la terapia del habla. Método: el levantamiento bibliográfico fue realizado en las bases de datos electrónicos: Pubmed, MEDLINE y Scielo, mediante la combinación de los siguientes Descriptores en Ciencias de la Salud (DeCs): "fisiología", "ejercicio"; "terapia miofuncional"; "disfagia" y "fonoaudiología", en los idiomas portugués y inglés. Resultados: En total, se encontraron 890 estudios en las bases de datos. Criterios de inclusión y exclusión se aplicaron en el título y en los resúmenes, siendo seleccionados 12 artículos, los cuales fueron analizados. Los artículos seleccionados presentaron qué músculos se activan durante la práctica de los ejercicios, cuál es la efectividad individual del ejercicio propuesto y los casos en que esas terapias son indicadas. Aunque estos estudios han contribuido a la base de conocimiento actual, poseen diferentes diseños metodológicos. Conclusión: Parece existir un número reducido de estudios que abordan los efectos promovidos por la TMO en los músculos y funciones orofaríngeas, siendo la mayoría de ellos relacionados con los estudios de la disfagia orofaríngea.

Palabras claves: Fisiología; Ejercicio; Orofaringe; Terapia Miofuncional; Fonoaudiología 


\section{Introdução}

Dentre as possíveis abordagens terapêuticas fonoaudiológicas para as regiões orofacial e orofaríngea, a utilização de exercícios constitui-se em proposta frequente para trazer mudanças na musculatura envolvida e na funcionalidade dessas regiões. A organização ou aprimoramento funcional da respiração, sucção, mastigação, articulação da fala, a partir de exercícios, visa promover resultados na musculatura orofaríngea que sejam protetores das vias aéreas, especialmente da deglutição. Exercícios que envolvem a musculatura orofaríngea, e consequentemente a deglutição, têm sido utilizados para buscar a modificação do comportamento muscular também durante o sono, a fim de reduzir os riscos de apneia obstrutiva do sono ${ }^{1}$.

Entretanto, pouco se procura compreender de que forma a realização de exercícios atua na modificação da musculatura e, principalmente, quais as mudanças que podem ser observadas a partir de um programa de treinamento com exercícios. Assim, como ponto de partida, parece decisivo compreender a morfologia e fisiologia do movimento muscular, assim como de exercícios específicos orofaríngeos ${ }^{2,3}$, como os apresentados na literatura referente à atuação com disfagia ${ }^{4}$.

O músculo estriado esquelético apresenta alta plasticidade, o que habilita este tecido alterar suas características morfológicas, metabólicas e funcionais em resposta a estímulos específicos. As características das fibras musculares e sua capacidade bioenergética para a produção de adenosina trifosfato (ATP), que fornece energia para abastecer a contração, determinam a força e a capacidade muscular ${ }^{5,6}$.

Os princípios do exercício relevantes à terapêutica fonoaudiológica são: intensidade, especificidade e transferência. Intensidade abrange a quantidade de carga, volume e duração de estímulo do exercício. Especificidade refere-se a quão próxima a tarefa de exercício corresponde ao resultado visado. Transferência baseia-se na lógica de usar treino cruzado e treino de força não específico para melhorar a função. Exercícios que não forçam o sistema neuromuscular além do nível da atividade habitual não provocarão adaptações, as adaptações só ocorrem ao desafiar o sistema além do uso típico ${ }^{7}$.

Especialistas em reabilitação devem conhecer os objetivos específicos atingidos com o exercício.
Descobertas na literatura sobre a ciência do exercício e reabilitação física sugerem que o método e a maneira de treinamento devem diferenciar significativamente se o objetivo é aumentar a força, a velocidade, a resistência ou alguma combinação deles. A compreensão de como o treinamento pode ser estruturado para facilitar e maximizar a plasticidade neuromuscular é um componente integral no desenvolvimento de tratamentos bem-sucedidos ${ }^{4}$.

Embora diversos autores apresentem programas de intervenção terapêutica fonoaudiológica de diferentes maneiras, pouco se compreende a respeito da morfofisiologia dos exercícios, isto é, apesar da literatura evidenciar a eficácia da Terapia Miofuncional Orofacial (TMO), os mecanismos pelos quais essas melhorias ocorrem são pouco elucidadas ${ }^{2,3,8-13}$

Considerando a necessidade de compreender os mecanismos pelos quais a terapia que se emprega é efetiva, o presente estudo busca analisar a literatura científica quanto à morfofisiologia dos exercícios orofaríngeos empregados na terapêutica fonoaudiológica.

\section{Métodos}

Trata-se de uma revisão integrativa seguindo seis fases de elaboração: identificação do tema e elaboração da hipótese ou pergunta norteadora, busca na literatura, coleta de dados, análise crítica dos estudos incluídos, discussão, interpretação dos resultados e apresentação da revisão integrativa ${ }^{14,15}$.

Como critério de busca foi utilizada a combinação dos seguintes descritores em Ciências da Saúde, nos idiomas inglês e português: "fisiologia" (physiology), "exercício" (exercise); "terapia miofuncional" (myofunctional therapy); "disfagia" (dysphagia) e "Fonoaudiologia" (Speech, Language and Hearing Sciences), por descritores associados (e/and).

Para o levantamento bibliográfico, foi realizada busca de artigos científicos nas bases de dados eletrônicas: "Medical Literature Analysis and Retrieval System Online" (MEDLINE), "US National Library of Medicine National Institutes Health" (Pubmed), Scientific Electronic Library Online (Scielo), sem restrição quanto à época de publicação. Foram obtidos 890 artigos considerando-se as três bases de dados. A partir desse número, o período de publicação foi restrito aos últimos cinco anos: período de janeiro de 2013 a novembro de 
2018, sendo também excluídos os títulos repetidos em mais de uma das bases de dados.

Os critérios de inclusão para a seleção dos artigos foram: artigos na íntegra disponíveis eletronicamente no momento do levantamento de dados publicados nos idiomas português ou inglês, que abordassem a musculatura ativa durante a prática dos exercícios orofaríngeos e a sua respectiva efetividade individual, assim como, os mecanismos pelos quais essa terapêutica é eficaz. Obrigatoriamente os estudos selecionados deveriam reportar dados morfofisiológicos dos exercícios orofaríngeos empregados.

A seleção de estudos iniciou-se a partir da verificação de títulos e resumos relacionados ao tema proposto, aplicação dos critérios de inclusão e de exclusão, leitura completa dos artigos e análise do material compondo o levantamento proposto. A apresentação dos dados foi efetuada de modo descritivo.

Para a coleta de dados e análise crítica dos estudos, foram utilizados dois instrumentos com informações sobre identificação, tipo de publicação, características metodológicas do estudo, resultados, além do exercício/terapia, musculatura ativa durante a terapêutica e efetividade individual da terapêutica.

\section{Resultados}

A partir do método utilizado, foram encontrados 890 estudos. A base de dados PUBMED foi a que mais publicou estudos relacionados com o tema, seguida da MEDLINE e SCIELO. Dentre os descritores as combinações: "fisiologia", "exercício" e "disfagia"; "fisiologia" "exercício" e "fonoaudiologia", foram as que possibilitaram encontrar maior número de estudos. A combinação dos descritores "fisiologia", "exercício" e "terapia miofuncional" foi a que propiciou menor número de resultados, conforme consta na Tabela 1. Esses dados mostram que o descritor "terapia miofuncional" é ainda pouco utilizado na literatura científica, uma vez que os estudos levantados com os descritores "fonoaudiologia" e "disfagia" apresentaram dados de terapia miofuncional.

A partir dos 890 estudos encontrados, 393 foram excluídos por restrição por período de publicação, 241 foram excluídos por duplicidade. Partindo-se, portanto, de 256 artigos inicialmente obtidos, apenas 12 contemplaram os critérios de inclusão para o presente estudo conforme diagrama de informações da seleção dos estudos encontrado na Figura 1.

Os artigos selecionados foram lidos na íntegra, avaliados conforme instrumento de coleta de dados e submetidos à análise crítica. Dentre os artigos, dez são artigos originais, um é relato de caso e um é artigo de revisão.

Os dados dos estudos encontram-se apresentadas no Quadro 1. Os exercícios referenciados nos artigos analisados estão apresentados no Quadro 2. A síntese dos dados extraídos do artigo de revisão da literatura encontra-se no Quadro 3.

Tabela 1. Estudos encontrados nas bases de dados com os descritores elencados.

\begin{tabular}{|c|c|c|c|}
\hline Descritores & PUBMED & MEDLINE & SCIELO \\
\hline $\begin{array}{l}\text { Fisiologia + exercício (physiology + exercise) + Terapia } \\
\text { Miofuncional (myofunctional therapy) }\end{array}$ & 33 & 0 & 0 \\
\hline $\begin{array}{l}\text { Fisiologia + exercício (physiology + exercise) + disfagia } \\
\text { (dysphagia) }\end{array}$ & 339 & 139 & 0 \\
\hline $\begin{array}{l}\text { Fisiologia + exercício (physiology + exercise) + } \\
\text { Fonoaudiologia (Speech, Language and Hearing Sciences). }\end{array}$ & 275 & 114 & 1 \\
\hline Total & 647 & 253 & 1 \\
\hline
\end{tabular}




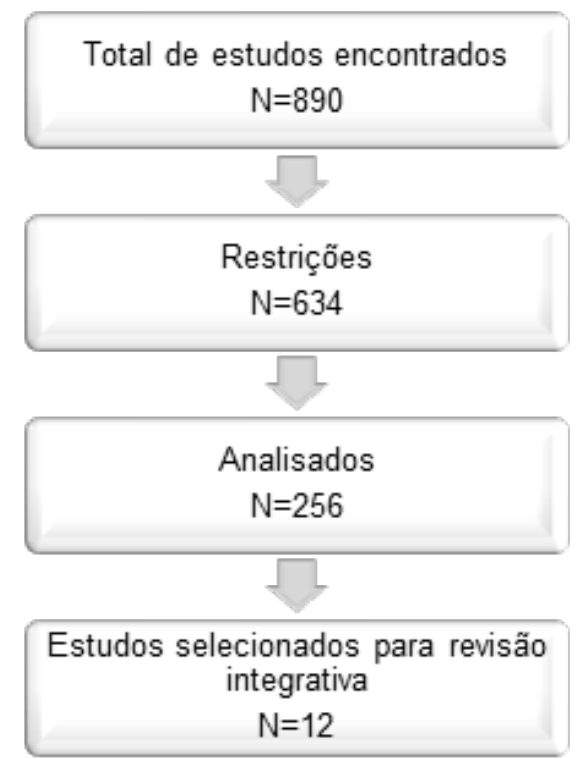

Figura 1. Etapas do processo de revisão de literatura

Quadro 1. Características dos estudos analisados.

\begin{tabular}{|c|c|c|c|c|c|}
\hline $\begin{array}{c}\text { Autor, Ano, } \\
\text { País }\end{array}$ & Estudo & Objetivos & Amostra & $\begin{array}{l}\text { Medidas dos } \\
\text { resultados }\end{array}$ & Resultados \\
\hline $\begin{array}{l}\text { Watts CR, } \\
2013^{(21)} \\
\text { EUA }\end{array}$ & $\begin{array}{l}\text { Prospectivo } \\
\text { Clínico }\end{array}$ & $\begin{array}{l}\text { Investigar os efei- } \\
\text { tos do exercício } \\
\text { de queixo contra } \\
\text { resistência em } \\
\text { comparação ao } \\
\text { exercício de ele- } \\
\text { vação de cabeça. }\end{array}$ & $\begin{array}{l}\text { Mulheres jovens } \\
\text { saudáveis (média } \\
\text { de idade: } 22,5 \\
\text { anos) }\end{array}$ & $\begin{array}{l}\text { Eletromiografia } \\
\text { de Superfície } \\
\text { (EMGs) }\end{array}$ & $\begin{array}{l}\text { A atividade dos mús- } \\
\text { culos laríngeos res- } \\
\text { ponsáveis por elevar } \\
\text { a laringe e movê-la } \\
\text { para a frente durante } \\
\text { a deglutição é maior } \\
\text { quando realizamos o } \\
\text { exercício de queixo } \\
\text { contra resistência. }\end{array}$ \\
\hline $\begin{array}{l}\text { Yoon WL et al, } \\
2014 \text { (22) } \\
\text { EUA }\end{array}$ & $\begin{array}{l}\text { Prospectivo } \\
\text { Clínico }\end{array}$ & $\begin{array}{l}\text { Comparar a ati- } \\
\text { vidade máxima e } \\
\text { média da EMGs } \\
\text { dos músculos su- } \\
\text { pra-hióideos du- } \\
\text { rante o exercício } \\
\text { de queixo contra } \\
\text { resistência e exer- } \\
\text { cício "Shaker" }\end{array}$ & $\begin{array}{l}40 \text { adultos } \\
\text { saudáveis (idade } \\
\text { entre } 21 \text { e } 39 \\
\text { anos) }\end{array}$ & $\begin{array}{l}\text { Eletromiografia } \\
\text { de Superfície } \\
\text { (EMGs) }\end{array}$ & $\begin{array}{l}\text { O exercício de queixo } \\
\text { contra resistência tem } \\
\text { impacto equivalente } \\
\text { ou maior ao exercício } \\
\text { "Shaker". }\end{array}$ \\
\hline $\begin{array}{l}\text { Fujiu-Kurachi } \\
\text { M et al, } \\
2014^{(27)} \\
\text { Japão }\end{array}$ & $\begin{array}{l}\text { Prospectivo } \\
\text { Clínico }\end{array}$ & $\begin{array}{l}\text { Investigar a } \\
\text { pressão intraoral } \\
\text { durante mano- } \\
\text { bra de Masako } \\
\text { em relação aos } \\
\text { diferentes graus } \\
\text { de protrusão da } \\
\text { língua }\end{array}$ & $\begin{array}{l}18 \text { jovens } \\
\text { saudáveis (média } \\
\text { de idade: } 26,9 \\
\text { anos) }\end{array}$ & $\begin{array}{l}\text { Sensor táctil } \\
\text { Swallow Scan } \\
\text { (Nitta, Osaka, } \\
\text { Japão) - cinco } \\
\text { locais no palato } \\
\text { duro }\end{array}$ & $\begin{array}{l}\text { A manobra de Masako } \\
\text { serve como exercício } \\
\text { de resistência que } \\
\text { coloca diferentes } \\
\text { quantidades de car- } \\
\text { ga nos músculos da } \\
\text { língua, alterando a } \\
\text { extensão da protrusão } \\
\text { da língua. }\end{array}$ \\
\hline $\begin{array}{l}\text { Shaker R et al, } \\
2016^{(25)} \\
\text { EUA }\end{array}$ & $\begin{array}{l}\text { Exploratório } \\
\text { descritivo }\end{array}$ & $\begin{array}{l}\text { Verificar se a } \\
\text { aplicação de re- } \\
\text { sistência contra o } \\
\text { movimento ân- } \\
\text { tero-superior do } \\
\text { complexo hiolarín- } \\
\text { geo irá sobrecar- } \\
\text { regar os músculos } \\
\text { faríngeos }\end{array}$ & $\begin{array}{l}30 \text { sujeitos } \\
\text { saudáveis } \\
\text { (idade entre } 42 \text { e } \\
56 \text { anos) }\end{array}$ & $\begin{array}{l}\text { Videofluoroscopia } \\
\text { da deglutição }\end{array}$ & $\begin{array}{l}\text { Repetidas deglutições } \\
\text { contra carga resistiva } \\
\text { induzida pela restrição } \\
\text { da excursão ântero- } \\
\text {-superior da laringe } \\
\text { induzem segurança na } \\
\text { fadiga do peristaltismo } \\
\text { faríngeo e, portanto, } \\
\text { tem potencial de for- } \\
\text { talecer a função con- } \\
\text { trátil da faringe. }\end{array}$ \\
\hline
\end{tabular}




\begin{tabular}{|c|c|c|c|c|c|}
\hline $\begin{array}{l}\text { Sze WP et al, } \\
2016^{(23)} \\
\text { EUA }\end{array}$ & $\begin{array}{l}\text { Prospectivo } \\
\text { Clínico }\end{array}$ & $\begin{array}{l}\text { Investigar a efi- } \\
\text { cácia do exercício } \\
\text { de queixo contra } \\
\text { resistência e } \\
\text { "Shaker", com } \\
\text { base nos princí- } \\
\text { pios de especifi- } \\
\text { cidade muscular } \\
\text { e intensidade de } \\
\text { treinamento }\end{array}$ & $\begin{array}{l}39 \text { adultos } \\
\text { saudáveis (média } \\
\text { de idade: } 29 \\
\text { anos) }\end{array}$ & $\begin{array}{l}\text { Eletromiografia } \\
\text { de Superfície } \\
\text { (EMGs) }\end{array}$ & $\begin{array}{l}\text { Exercício de queixo } \\
\text { contra resistência } \\
\text { causa impacto maior } \\
\text { nos músculos supra- } \\
\text {-hióideos, e menor } \\
\text { em músculos não } \\
\text { alvo comparado ao } \\
\text { "Shaker". }\end{array}$ \\
\hline $\begin{array}{l}\text { Park JS et al, } \\
2016^{(19)} \\
\text { Coreia }\end{array}$ & $\begin{array}{l}\text { Clínico } \\
\text { Controlado } \\
\text { Randomizado }\end{array}$ & $\begin{array}{l}\text { Investigar efeitos } \\
\text { do treinamento } \\
\text { muscular expira- } \\
\text { tório na atividade } \\
\text { dos músculos } \\
\text { supra-hióideos, } \\
\text { aspiração e dieté- } \\
\text { tica em pacientes } \\
\text { com AVC e dis- } \\
\text { fagia. }\end{array}$ & $\begin{array}{l}33 \text { pacientes } \\
\text { acometidos por } \\
\text { AVC com disfagia } \\
\text { (média de idade: } \\
64,3 \text { ) }\end{array}$ & $\begin{array}{l}\text { Eletromiografia } \\
\text { de Superfície } \\
\text { (EMGs) } \\
\text { Videofluoroscopia } \\
\text { da deglutição. }\end{array}$ & $\begin{array}{l}\text { O treinamento de } \\
\text { força muscular ex- } \\
\text { piratória é eficaz em } \\
\text { estimular a atividade } \\
\text { no grupo de múscu- } \\
\text { los supra-hióideos de } \\
\text { pacientes com disfagia } \\
\text { após um acidente } \\
\text { vascular cerebral. }\end{array}$ \\
\hline $\begin{array}{l}\text { Kraaijenga SAC } \\
\text { et al, } 2016^{(18)} \\
\text { Amsterdam }\end{array}$ & $\begin{array}{l}\text { Prospectivo } \\
\text { Clínico }\end{array}$ & $\begin{array}{l}\text { Investigar eficácia } \\
\text { de um protocolo } \\
\text { de treinamento } \\
\text { intensivo de for- } \\
\text { ça em pacientes } \\
\text { com câncer de } \\
\text { cabeça e pescoço } \\
\text { e disfagia após } \\
\text { tratamento com } \\
\text { radioterapia }\end{array}$ & $\begin{array}{l}17 \text { pacientes } \\
\text { com câncer de } \\
\text { cabeça e pescoço } \\
\text { e disfagia, (média } \\
\text { de idade: } 65 \\
\text { anos) }\end{array}$ & $\begin{array}{l}\text { Videofluoroscopia } \\
\text { da deglutição }\end{array}$ & $\begin{array}{l}\text { Efeitos objetivos e } \\
\text { subjetivos da carga } \\
\text { progressiva na força } \\
\text { muscular e na função } \\
\text { de deglutição foram } \\
\text { demonstrados, indi- } \\
\text { cando que os mús- } \\
\text { culos da deglutição a } \\
\text { longo prazo ainda são } \\
\text { treináveis. }\end{array}$ \\
\hline $\begin{array}{l}\text { Namasivayam- } \\
\text { MacDonald AM } \\
\text { et al, 2017 (26) } \\
\text { Canadá }\end{array}$ & $\begin{array}{l}\text { Série de } \\
\text { casos }\end{array}$ & $\begin{array}{l}\text { Determinar eficá- } \\
\text { cia do treinamen- } \\
\text { to de pressão de } \\
\text { língua em idosos } \\
\text { com comprome- } \\
\text { timento cognitivo } \\
\text { leve a grave no } \\
\text { cenário de cuida- } \\
\text { dos a longo prazo } \\
\end{array}$ & $\begin{array}{l}\text { Idosos com } \\
\text { comprometimento } \\
\text { cognitivo leve a } \\
\text { grave (média de } \\
\text { idade: } 91 \text { anos) }\end{array}$ & $\begin{array}{l}\text { Iowa Oral } \\
\text { Performance } \\
\text { Instrument } \\
\text { (IOPI) }\end{array}$ & $\begin{array}{l}\text { Houve clara diferença } \\
\text { entre pressões iniciais } \\
\text { e finais da língua. No } \\
\text { entanto, apesar de } \\
\text { melhoras em pressões } \\
\text { de língua, não iden- } \\
\text { tificou-se melhoras } \\
\text { associadas a função } \\
\text { da deglutição. }\end{array}$ \\
\hline $\begin{array}{l}\text { Hutcheson KA } \\
\text { et al, } 2017(24) \\
\text { EUA }\end{array}$ & $\begin{array}{l}\text { Relatório } \\
\text { Técnico }\end{array}$ & $\begin{array}{l}\text { Detectar padrões } \\
\text { relacionados à de- } \\
\text { glutição e ativida- } \\
\text { de dos músculos } \\
\text { palatino, laríngeo } \\
\text { e faríngeo durante } \\
\text { o treinamento } \\
\text { expiratório }\end{array}$ & $\begin{array}{l}2 \text { mulheres } \\
\text { saudáveis (idade } \\
23 \text { e } 24 \text { anos) }\end{array}$ & $\begin{array}{l}\text { Manometria } \\
\text { da faringe de } \\
\text { alta resolução } \\
\text { Eletromiografia } \\
\text { de superfície e } \\
\text { intramuscular. }\end{array}$ & $\begin{array}{l}\text { Os mecanismos pelos } \\
\text { quais o treinamento } \\
\text { de força muscular } \\
\text { expiratória pode me- } \\
\text { Ihorar a proteção das } \\
\text { vias aéreas relacio- } \\
\text { nadas à deglutição } \\
\text { provavelmente envol- } \\
\text { ve músculos além da } \\
\text { região supra hioidea e } \\
\text { laríngea. }\end{array}$ \\
\hline $\begin{array}{l}\text { Eom MJ et al, } \\
2017_{(20)} \\
\text { Coreia }\end{array}$ & $\begin{array}{l}\text { Clínico } \\
\text { randomizado }\end{array}$ & $\begin{array}{l}\text { Investigar o efeito } \\
\text { do treinamento } \\
\text { de força muscular } \\
\text { expiratória na } \\
\text { função da degluti- } \\
\text { ção em pacientes } \\
\text { com AVC e disfa- } \\
\text { gia orofaríngea. }\end{array}$ & $\begin{array}{l}42 \text { pacientes com } \\
\text { AVC e disfagia } \\
\text { (média de idade: } \\
69,2 \text { ) }\end{array}$ & $\begin{array}{l}\text { Videofluoroscopia } \\
\text { da deglutição }\end{array}$ & $\begin{array}{l}\text { O treinamento de } \\
\text { força muscular expi- } \\
\text { ratória pode melhorar } \\
\text { os efeitos da disfagia } \\
\text { em pacientes idosos } \\
\text { após AVC com base na } \\
\text { função de deglutição. }\end{array}$ \\
\hline $\begin{array}{l}\text { Park JS et al, } \\
2017(17) \\
\text { Coreia }\end{array}$ & $\begin{array}{l}\text { Clínico não } \\
\text { randomizado }\end{array}$ & $\begin{array}{l}\text { Investigar o efeito } \\
\text { do exercício de } \\
\text { elevação de ca- } \\
\text { beça no complexo } \\
\text { laríngeo e aspira- } \\
\text { tivo em pacientes } \\
\text { com AVC e dis- } \\
\text { fagia }\end{array}$ & $\begin{array}{l}27 \text { pacientes } \\
\text { acometidos por } \\
\text { AVC disfágicos } \\
\text { (média de idade: } \\
59,26 \text { ) }\end{array}$ & $\begin{array}{l}\text { Videofluoroscopia } \\
\text { da deglutição }\end{array}$ & $\begin{array}{l}\text { O exercício de ele- } \\
\text { vação de cabeça é } \\
\text { eficaz para melhorar o } \\
\text { movimento do hioide e } \\
\text { diminuir aspiração em } \\
\text { pacientes disfágicos } \\
\text { acometido por AVC. }\end{array}$ \\
\hline
\end{tabular}


Quadro 2. Dados dos exercícios apresentados nos estudos selecionados

\begin{tabular}{|c|c|c|c|}
\hline Autor, Ano, País & Exercício & $\begin{array}{l}\text { Musculatura ativa } \\
\text { durante exercício }\end{array}$ & $\begin{array}{l}\text { Efetividade individual do } \\
\text { programa }\end{array}$ \\
\hline $\begin{array}{l}\text { Watts CR, } 2013^{(21)} \\
\text { EUA }\end{array}$ & $\begin{array}{l}\text { Dispositivo semirrígido } \\
\text { ajustável. } \\
\text { "Exercício de queixo contra } \\
\text { resistência" } \\
\text { "Exercício de elevação de } \\
\text { cabeça" }\end{array}$ & Músculos supra-hióideos & $\begin{array}{l}\text { Melhorar excursão hiolaríngea e } \\
\text { abertura do EES }\end{array}$ \\
\hline $\begin{array}{l}\text { Yoon WL et al, } \\
2014^{(22)} \\
\text { EUA }\end{array}$ & $\begin{array}{l}\text { "Exercício de queixo contra } \\
\text { a resistência" e "Exercício } \\
\text { Shaker" }\end{array}$ & Músculos supra-hióideos & $\begin{array}{l}\text { Promover o movimento anterior } \\
\text { e superior da laringe e osso } \\
\text { hióide, resultando em abertura } \\
\text { do EES. }\end{array}$ \\
\hline $\begin{array}{l}\text { Fujiu-Kurachi M et } \\
\text { al, } 2014 \text { (27) } \\
\text { Japão }\end{array}$ & "Manobra de Masako" & $\begin{array}{l}\text { Base da língua e parede } \\
\text { faríngea }\end{array}$ & $\begin{array}{l}\text { Facilitar a constrição faríngea } \\
\text { durante o estágio faríngeo da } \\
\text { deglutição. }\end{array}$ \\
\hline $\begin{array}{l}\text { Shaker R et al, } \\
2016^{(25)} \\
\text { EUA }\end{array}$ & $\begin{array}{l}\text { Dispositivo artesanal que } \\
\text { afeta "excursão laríngea } \\
\text { induzida pela deglutição e } \\
\text { abertura EES" }\end{array}$ & $\begin{array}{l}\text { Músculos supra-hióideos e } \\
\text { constritores faríngeos }\end{array}$ & $\begin{array}{l}\text { A resistência contra o } \\
\text { movimento ântero-superior } \\
\text { do complexo laríngeo durante } \\
\text { a deglutição sobrecarrega os } \\
\text { músculos faríngeos e, pela } \\
\text { deglutição repetitiva, resultar } \\
\text { em fadiga neuromuscular. } \\
\end{array}$ \\
\hline $\begin{array}{l}\text { Sze WP et al, } \\
2016^{(23)} \\
\text { EUA }\end{array}$ & $\begin{array}{l}\text { "Exercício de queixo contra } \\
\text { resistência" "Exercício } \\
\text { Shaker" }\end{array}$ & $\begin{array}{l}\text { Músculos supra- } \\
\text { hióideos, infra-hióideos, } \\
\text { escaleno anterior e } \\
\text { esternocleidomastoideo }\end{array}$ & $\begin{array}{l}\text { Manter a patência da via aérea } \\
\text { e a posição do hioide. } \\
\text { Aumentar diâmetro } \\
\text { anteroposterior da abertura } \\
\text { do EES e a excursão laríngea } \\
\text { anterior máxima pós-exercício. }\end{array}$ \\
\hline $\begin{array}{l}\text { Park JS et al, } \\
2016_{(19)} \\
\text { Coreia }\end{array}$ & $\begin{array}{l}\text { "Treinamento de força } \\
\text { muscular expiratória" }\end{array}$ & $\begin{array}{l}\text { Músculos digástricos, } \\
\text { milo-hioídeo e gênio- } \\
\text { hióideo. }\end{array}$ & $\begin{array}{l}\text { Melhorar sistema ventilatório, } \\
\text { via fortalecimento dos músculos } \\
\text { respiratórios através da ação } \\
\text { expiratória oral forte. }\end{array}$ \\
\hline $\begin{array}{l}\text { Kraaijenga SAC et } \\
\text { al, 2016(18) } \\
\text { Amsterdam }\end{array}$ & $\begin{array}{l}\text { Dispositivo } \\
\text { "Exercício de queixo contra a } \\
\text { resistência" } \\
\text { "Exercício de abertura } \\
\text { mandibular contra resistência" } \\
\text { "Esforço de deglutição" }\end{array}$ & $\begin{array}{l}\text { Músculos supra-hióideos e } \\
\text { faríngeos }\end{array}$ & $\begin{array}{l}\text { Melhorar elevação hiolaríngea } \\
\text { e abertura do EES. Aumentar } \\
\text { a retração da base da língua e } \\
\text { diminuir quantidade de resíduo } \\
\text { faríngeo }\end{array}$ \\
\hline $\begin{array}{l}\text { Namasivayam- } \\
\text { MacDonald AM et al, } \\
2017 \text { (26) } \\
\text { Canada }\end{array}$ & Treinamento lingual & $\begin{array}{l}\text { Região anterior e } \\
\text { posterior de língua }\end{array}$ & Melhorar força lingual \\
\hline $\begin{array}{l}\text { Hutcheson KA et al, } \\
2017^{(24)} \\
\text { EUA }\end{array}$ & $\begin{array}{l}\text { Treinamento de força } \\
\text { muscular expiratória }\end{array}$ & $\begin{array}{l}\text { Músculos supra-hióideos, } \\
\text { velofaríngeos e laríngeos }\end{array}$ & $\begin{array}{l}\text { Estabelecer encurtamento } \\
\text { faríngeo vertical à medida que } \\
\text { a estrutura laríngea se eleva } \\
\text { durante a tarefa expiratória } \\
\text { e / ou redução ativa da área } \\
\text { transversal faríngea à medida } \\
\text { que a via aérea se comprime } \\
\text { durante as tarefas expiratórias }\end{array}$ \\
\hline $\begin{array}{l}\text { Eom MJ et al, } \\
2017(20) \\
\text { Coréia }\end{array}$ & $\begin{array}{l}\text { Treinamento de força } \\
\text { muscular expiratória }\end{array}$ & Músculos supra-hióideos & $\begin{array}{l}\text { Propiciar movimento superior } \\
\text { e anterior do osso hioide e da } \\
\text { laringe estabelecendo efeitos } \\
\text { diretos na aspiraçãa, abertura } \\
\text { do EES e resíduo faríngeo. } \\
\text { Estimular o centro de deglutição } \\
\text { no tronco encefálico. }\end{array}$ \\
\hline $\begin{array}{l}\text { Park JS et al, } \\
2017(17) \\
\text { Coréia }\end{array}$ & $\begin{array}{l}\text { Exercício de elevação de } \\
\text { cabeça (EHL) }\end{array}$ & $\begin{array}{l}\text { Músculos digástricos, } \\
\text { gênio-hióideo e milo- } \\
\text { hioídeo }\end{array}$ & $\begin{array}{l}\text { Propiciar abertura do EES, } \\
\text { auxiliando na prevenção de } \\
\text { aspiração enquanto o bolo } \\
\text { passa para a faringe }\end{array}$ \\
\hline
\end{tabular}


Quadro 3. Síntese da apresentação do artigo de revisão de literatura (Hegland \& Murry, 2013'6)

\begin{tabular}{|c|c|c|c|c|c|c|}
\hline \multicolumn{7}{|c|}{ Autor, Ano, País } \\
\hline \multicolumn{7}{|c|}{ Hegland e Murry, $2013^{(16)}$, EUA } \\
\hline Exercícios & $\begin{array}{l}\text { Deglutição } \\
\text { supra glótica }\end{array}$ & $\begin{array}{l}\text { Manobra de } \\
\text { esforço da } \\
\text { deglutição }\end{array}$ & $\begin{array}{l}\text { Manobra de } \\
\text { Mendelson }\end{array}$ & $\begin{array}{c}\text { Fortalecimento } \\
\text { da língua }\end{array}$ & 'Shaker" & $\begin{array}{l}\text { Treinamento } \\
\text { de força } \\
\text { muscular } \\
\text { expiratória. }\end{array}$ \\
\hline $\begin{array}{l}\text { Musculatura } \\
\text { ativa durante } \\
\text { o exercício }\end{array}$ & Pregas vocais & $\begin{array}{l}\text { Base da língua } \\
\text { e músculos da } \\
\text { faringe }\end{array}$ & $\begin{array}{l}\text { Laringe } \\
\text { (elevação) }\end{array}$ & $\begin{array}{c}\text { Região } \\
\text { anterior e } \\
\text { posterior da } \\
\text { língua }\end{array}$ & $\begin{array}{c}\text { Músculos } \\
\text { gênio-hioideo, } \\
\text { tireo-hioideo, } \\
\text { digástricos }\end{array}$ & $\begin{array}{c}\text { Músculos } \\
\text { expiratórios, } \\
\text { supra-hióideos } \\
\text { e laríngeos }\end{array}$ \\
\hline $\begin{array}{c}\text { Efetividade } \\
\text { individual do } \\
\text { programa }\end{array}$ & $\begin{array}{l}\text { Limpar } \\
\text { resíduos do } \\
\text { vestíbulo } \\
\text { laríngeo }\end{array}$ & $\begin{array}{l}\text { Impulsionar } \\
\text { o bolo para } \\
\text { dentro da } \\
\text { orofaringe } \\
\text { Redução ou } \\
\text { eliminação } \\
\text { de resíduo } \\
\text { faríngeo. }\end{array}$ & $\begin{array}{l}\text { Abrir o EES } \\
\text { prolongando } \\
\text { a duração } \\
\text { da elevação } \\
\text { laríngea }\end{array}$ & $\begin{array}{l}\text { Diminuir a } \\
\text { duração do } \\
\text { trânsito oral e } \\
\text { aumentar } \\
\text { pressões de } \\
\text { deglutição }\end{array}$ & $\begin{array}{c}\text { Fortalecer } \\
\text { os músculos } \\
\text { gênio- } \\
\text { hioideos, } \\
\text { tireo-hioideos } \\
\text { e digástricos. } \\
\text { Diminuir } \\
\text { a pressão } \\
\text { do bolo } \\
\text { hipofaríngeo } \\
\text { ao entrar no } \\
\text { EES. }\end{array}$ & $\begin{array}{c}\text { Aumentar a } \\
\text { força muscular } \\
\text { expiratória } \\
\text { Aumentar } \\
\text { a ativação } \\
\text { supra hioidea } \\
\text { anterior. } \\
\text { Aumentar o } \\
\text { movimento da } \\
\text { laringe }\end{array}$ \\
\hline
\end{tabular}

\section{Discussão}

Os estudos verificados no presente estudo analisaram especificamente a aplicabilidade e resultados da TMO em adultos e em idosos saudáveis; em acometidos por Acidente Vascular Cerebral (AVC); em câncer de cabeça e pescoço e naqueles com comprometimento cognitivo. A revisão de literatura selecionada ${ }^{16}$ apresenta os tipos de tratamentos não cirúrgico para reabilitação da deglutição. Parece importante notar que os estudos que apontam dados da morfofisiologia de exercícios orofaríngeos são aqueles que enfocam os quadros de disfagia ${ }^{16-24}$.

As terapêuticas elegíveis nos estudos revisados foram: "Shaker" ou elevação de cabeça; exercício de queixo contra resistência; esforço de deglutição; exercício de abertura mandibular contra resistência, treinamento de força muscular expiratória; fortalecimento de língua; exercício de resistência a deglutição. Além destes exercícios a revisão de literatura selecionada ${ }^{16}$ apresentou mais dois exercícios: deglutição supraglótica e manobra de Mendelson, que não foram encontrados nos artigos originais incluídos neste estudo.

O ano de publicação da revisão de literatura selecionada ${ }^{16}$ é 2013 , logo é possível supor que, de acordo com os critérios de inclusão deste estudo, nos últimos anos pouco se tem investigado sobre a deglutição supraglótica e manobra de Mendelson.
Dentre os exercícios investigados nos estudos revisados, houve predomínio na descrição e proposta de intervenção do exercício de queixo contra resistência, "Shaker" e treinamento de força muscular expiratória.

Vale ressaltar que, Hegland e Murry ${ }^{16}$ em sua revisão de literatura apresentam o exercício de elevação de cabeça também descrito como "Shaker", como o exercício que propicia o recrutamento do músculo gênio-hióideo, tireo-hióideo e digástricos. $\mathrm{O}$ fortalecimento desse grupo muscular, favorece a excursão hiolaríngea e abertura do Esfíncter Esofágico Superior (EES) ${ }^{16}$.

Park JS et al ${ }^{17}$ ao investigar o efeito do exercício de elevação de cabeça ("Shaker) no complexo laríngeo e aspirativo de pacientes acometidos por acidente vascular cerebral disfágicos, propõem que este exercício se caracteriza como intervenção indireta, segura e eficaz para melhorar o movimento anterior e superior do osso hióide contribuindo para a abertura do EES auxiliando na prevenção de aspiração.

Em outro estudo ${ }^{18}$ com uma amostra de pacientes com câncer de cabeça e pescoço, foram aplicados os exercícios: queixo contra a resistência, abertura mandibular contra resistência e esforço da deglutição. Segundo os autores, o exercício de abertura mandibular contra resistência e esforço da deglutição favorecem o aumento da retração da base da língua e diminuição da quantidade de 
resíduo faríngeo, enquanto que o exercício de queixo contra a resistência tem a mesma finalidade do exercício "Shaker", isto é, a melhora da elevação hiolaríngea e a abertura do EES, conforme descritos anteriormente ${ }^{16,17}$.

Três estudos diferentes ${ }^{21-23}$ utilizaram sujeitos saudáveis e o mesmo instrumento de avaliação: Eletromiografia de Superfície (EMGs), com o objetivo de comparar os efeitos do exercício "Shaker" e exercício de queixo contra resistência como proposta de intervenção para disfagia.

Os resultados obtidos por Watts ${ }^{21}$ mostraram que a atividade dos músculos responsáveis por elevar a laringe e movê-la para a frente durante a deglutição é maior quando se realiza o exercício de queixo contra resistência em comparação ao exercício "Shaker".

Da mesma forma, Yoon et $\mathrm{al}^{22}$ propõem que o exercício de queixo contra a resistência resultou em ativação significativamente maior durante a tarefa isocinética e isométrica sobre os músculos supra-hióideos, concluindo que o exercício de queixo contra a resistência tem impacto equivalente ou maior ao exercício "Shaker".

Ao se referir a estas terapêuticas Sze WP et al ${ }^{23}$ relataram que o exercício de queixo contra resistência além de causar impacto significativamente maior nos músculos supra-hióideos apresenta maior precisão no direcionamento dos músculos supra-hióideos, uma vez que a ativação de músculos não-alvo é uma consequência da dinâmica do exercício "Shaker".

Esses achados sugerem duas possibilidades de exercícios para promover a excursão hiolaríngea e abertura do EES, apresentando o exercício de queixo contra resistência como o mais promissor. Os estudos ${ }^{21-23}$ foram publicados em 2013, 2014 e 2016 sugerindo que essa teoria se mantém nos últimos anos.

Estudos também relataram a eficácia do treinamento de força muscular expiratória. Segundo os autores ${ }^{16} \mathrm{o}$ treinamento de força muscular expiratória proporciona o recrutamento da musculatura expiratória, supra-hióidea e laríngea.

Os resultados obtidos em um Estudo Clínico Randomizado ${ }^{19}$ (ECR) mostraram que o treinamento de força muscular expiratória propicia atividade no grupo muscular supra-hióideo e contribui para diminuição da aspiração em pacientes com AVC e disfagia. Outro estudo ${ }^{20}$ estabeleceu a mesma metodologia, e os resultados corroboram com o estudo anterior $^{19}$ de que a forte contração dos músculos supra-hióideos afeta diretamente o movimento superior e anterior do osso hióide e da laringe. Acrescentam ainda que, durante o treinamento de força muscular expiratória, os receptores sensoriais da língua e orofaringe aumentam em vários estímulos aferentes, desencadeando a ativação do centro da deglutição localizado na medula oblonga do tronco encefálico.

Estudo não randomizado ${ }^{24} \mathrm{com}$ desenho metodológico diferente dos anteriores ${ }^{19,20}$ refere, que durante as tarefas expiratórias a atividade da musculatura faríngea pode refletir encurtamento faríngeo vertical à medida que a estrutura laríngea se eleva ou redução da área transversal faríngea, à medida que a via aérea se comprime durante as tarefas expiratórias. Os autore ${ }^{24}$ também observaram que os músculos supra-hióideos e o músculo tireoaritenóideo parecem estar mais ativos antes da tarefa expiratória, isto é, no ato de preparação para execução da tarefa expiratória que durante a tarefa em si. Concluindo, que os mecanismos pelos quais o treinamento de força muscular expiratória melhora a proteção das vias aéreas durante à deglutição, envolve músculos além da região supra-hióidea e laríngea.

Os achados sobre a deglutição supra glótica e manobra de Mendelson foram encontrados apenas em uma revisão de literatura ${ }^{16}$. Esta revisão aponta que a manobra de Mendelson propicia a elevação laríngea ocasionando a abertura do EES e que a deglutição supra glótica fecha as pregas vocais durante a deglutição, favorecendo a limpeza de resíduos no vestíbulo laríngeo. De acordo com os estudos incluídos não encontramos artigos originais para confirmar tais descrições.

Com a hipótese de promover resistência a deglutição por meio de um dispositivo artesanal que afeta a excursão laríngea, Shaker et $\mathrm{al}^{25}$ apresentam que a deglutição repetitiva contra aumento de carga resulta em fadiga neuromuscular. Este resultado sugere estudos futuros para avaliar diretamente a melhoria potencial em força muscular faríngea e aumento da pressão faríngea.

Namasivayam-MacDonald et $\mathrm{al}^{26}$, analisando a eficácia do treinamento de pressão de língua em idosos com comprometimento cognitivo leve a moderadamente grave, mostraram clara diferença entre as pressões iniciais e finais da língua com a utilização do Iowa Oral Performance Instrument (IOPI) no entanto, apesar dessas melhorias nas 
pressões de língua, não foram identificadas melhorias associadas à função da deglutição.

Fujiu-Kurachi et al $^{27}$ ao investigar a pressão intraoral durante manobra de Masako em relação aos diferentes graus de protrusão da língua concluem que essa serve como exercício de resistência que, alterando a extensão da protrusão da língua coloca diferentes quantidades de carga nos músculos da língua. Os autores relataram que a extensão máxima da protrusão da língua de um indivíduo deve ser levada em consideração, pois essa característica pode restringir o movimento da língua.

Os artigos selecionados apresentaram quais músculos são ativados durante a prática dos exercícios, qual efetividade individual do exercício proposto e os casos em que essas terapêuticas são indicadas. Embora esses estudos tenham contribuído para a base de conhecimento atual eles possuem diferentes desenhos metodológicos. Dentre os artigos selecionados, apenas dois ${ }^{19,20}$ caracterizam-se como ECR e apenas sete estudos ${ }^{16,18,21-23,25,27}$ discutiram sobre os princípios do exercício.

As principais limitações desses estudos ${ }^{16-27}$ são relacionadas ao tamanho de amostra reduzido; aos efeitos da recuperação natural pela neuroplasticidade; a não apresentação de exames de imagem cerebral uma vez que a adaptação neural relacionada à deglutição não pôde ser firmemente confirmada; e por fim, não houve acompanhamento após o término da intervenção e a durabilidade dos efeitos não pôde ser determinada.

Não foram encontrados estudos de pesquisadores brasileiros descrevendo a morfofisiologia do exercício orofaríngeo. Grande parte dos estudos tem descrito a eficácia de programas terapêuticos, considerando apenas seus efeitos sem especificar se os exercícios empregados no programa são eficientes individualmente e qual a maneira de realização que garantiria o alcance dos objetivos propostos.

$\mathrm{Na}$ maioria dos estudos revisados ${ }^{17-23,25}$, os autores utilizaram como medida dos resultados a Eletromiografia de Superfície (EMGs) e a videofluoroscopia da deglutição. Três estudos ${ }^{24,26,27}$ utilizaram instrumentos diferentes, sendo eles o Iowa Oral Performance Instrument (IOPI); Sensor táctil Swallow Scan; Manometria Faríngea e Eletromiografia Intramuscular. Todos os dados relatados dizem respeito ao efeito da terapia imediatamente após a conclusão da TMO, e os relatos sobre avaliação miofuncional podem ser compreendidos como limitados.
A maioria dos autores revisados ${ }^{16-25}$ apresentou como resultados da TMO a adequação da musculatura supra-hióidea, porém alguns dos métodos utilizados podem refletir o comportamento de musculatura próxima ou associada aos supra-hióideos, sendo, portanto, de difícil conclusão.

\section{Conclusão}

Parece existir reduzido número de estudos que abordem os efeitos promovidos pela TMO nos músculos e funções orofaríngeas, sendo a maioria deles relacionados aos estudos da disfagia orofaríngea. Aspectos referentes à morfofisiologia são abordados especialmente quanto a exercícios específicos voltados para a musculatura supra-hióidea e laríngea. Pouco se discute se os efeitos da TMO estão, de fato, relacionados à melhora das funções musculares e orofaciais.

\section{Referências}

1. Kayamori F, Bianchini EMG. Effects of orofacial myofunctional therapy on the symptoms and physiological parameters of sleep breathing disorders in adults: a systematic review. Rev. CEFAC. 2017 Dec; 19 (6): 868-78.

2. Steele CM. On the plausibility of upper airway remodeling as an outcome of orofacial exercise. Am J Respir Crit Care Med. 2009; 179(10): 858-9.

3. Ferreira TS, Mangilli LD, Sassi FC, Tavares TF, Limongi SCO, Andrade CRF. Fisiologia do exercício fonoaudiológico: uma revisão crítica da literatura. J Soc Bras Fonoaudiol. 2011; 23 (3): 288-96.

4. Burkhead LM, Sapienza CM, Rosenbek JC. Strength-training exercise in dysphagia rehabilitation: principles, procedures and directions for future research. Dysphagia. $2007 \mathrm{Jul} ; 22$ (3): 251-65.

5. Seene T, Kaasik P. Biological Characteristics of Structural and Functional Remodelling in Skeletal Muscle: Effect of Exercise. Advanced Studies in Biology:2013; 5(6): 251-78

6. Aguiar AF, Aguiar DH. Plasticidade muscular no exercício físico. R. bras. Ci. e Mov. 2009; 17 (3): 104-13.

7. Crary MA, Carnaby GD. Adoption into clinical practice of two therapies to manage swallowing disorders: exercise-basede swallowing rehabilitation and electrical stimulation. Curr Opin Otolaryngol Head Neck Surg. 2014 Jun; 22(3): 172-80.

8. Langmore SE, Pisegna JM. Efficacy of exercises to rehabilitate dysphagia: A critique of the literature. Int J Speech Lang Pathol. 2015 Jun;17 (3): 222-9.

9. Andrade CRF, Sassi FC, Juste FS, Ercolin B. Modelamento da fluência com o uso da eletromiografia de superfície: estudo piloto. Revista de Atualização Científica. 2008; 20 (2): 129-32.

10. Marson A, Tessitore A, Sakano E, Nemr K. Efetividade da fonoterapia e proposta de intervenção breve em respiradores orais. Rev. CEFAC. 2012; 14 (6): 1153-66. 
11. Diaféria G, Badke L, Silva RS, Bommarito S, Tufik S, Bittencourt L. Effect of speech therapy as adjunct treatment to continuous positive airway pressure on the quality of life of patients with obstructive sleep apnea. Sleep Med. 2013; 14 (7): 628-35.

12. Rahal A. Bases da terapia de motricidade orofacial. In: Tessitore A, Marchesan IQ, Justino H, Berretin-Felix G. Práticas Clínicas em Motricidade Orofacial. $1^{\text {a }}$ ed. Pinhais: Melo, 2014, p. 147-52.

13. Migliorucci RR, Passos DCBOF, Berretin-Felix G. Programa de terapia miofuncional orofacial para indivíduos submetidos à cirurgia ortognática. Rev. CEFAC. 2017; 19(2): 277-88.

14. Mendes KDS, Silveira RCCP, Galvão CM. Revisão integrativa: método de pesquisa para a incorporação de evidências na saúde e na enfermagem. Texto Contexto Enferm. 2008; 17 (4): 758-64.

15. Souza MT, Silva MD, Carvalho R. Revisão integrativa: o que é e como fazer. Einstein. 2010 Jun; 8 (1): 102-6.

16. Hegland KW, Murry T. Nonsurgical treatment: swallowing rehabilitation. Otolaryngol Clin North Am. 2013 Dec; 46 (6): 1073-85.

17. Park JS, Hwang M, Chang M. Effect of head lift exercise on kinematic motion of the hyolaryngeal complex and aspiration in patients with dysphagic stroke. J Oral Rehabil. 2017 May; 44(5): 385-91.

18. Kraaijenga SAC, Stuiver MM, Al-Mamgani A, Hilgers FJM, Molen LVD, Takes RP, et al. Efficacy of a novel swallowing exercise program for chronic dysphagia in long-term and neck cancer surviuors. Head Neck. 2017 Oct; 39(10): 1943-61.

19. Park JS, Oh DH, Chang MY, Kim KM. Effects of expiratory muscle strength training on oropharyngeal dysphagia in subacute stroke patients: a randomized controlled trial. J Oral Rehabil. 2016 May; 43(5): 364-72.
20. Eom MJ, Chang LJ, Hwan DO, Kim HD, Han NM, Park JS. Efeitos do treinamento resistido efeitos de força muscular expiratória em pacientes idosos com acidente vascular cerebral disfágicos. Neuro Reabilitação. 2017; 41 (4): 747-52

21. Watts CR. Measurement of Hyolaryngeal Muscle Activation Using Surface Electromyography for Comparison of Two Rehabilitative Dysphagia Exercise. Arch Phy Med Rehabil. 2013 Dec; 94(12): 2542-8.

22. Yoon WL, Khoo JK, Rickard Liow SJ. Chin tuck against resistance (CTAR): new method for enhancing suprahyoid muscle activityusing a Shaker-type exercise. Dysphagia, 2014 Apr; 29(2): 243-8.

23. Sze WP, Yoon WL, Escoffier N, Liow SJR. Evaluating the Training Effects of two swallowing rehabilitation therapies using surface etectromyography- Chin Tuck Against Resistance (CTAR) Exercise and the Shaker Exercise. Dysphagia. 2016 Apr; 31 (2): 195-205.

24. Hutcheson KA, Hammer MJ, Rosen SP, Jones CA, Culloch TM. Expiratory muscle strength training evaluated with simultaneous high-resolution manometry and electromyography. Laryngoscopy, 2017 Apr; 127 (4): 797-804.

25. Shaker R, Sanvanson P, Balasubramanian G, Kern M, Wuerl A, Hyngstrom A. Effects of laryngeal restriction on pharyngeal peristalsis and biomechanics: Clinical implications. Am J Physiol Gastrointest Liver Physiol. 2016 Jun; 310 (11): 1036-43.

26. Namasivayam-MacDonald AM, Burnett L, Nagy A, Waito AA, Steele CM. Effects of tongue Strength Training on Mealtime function in long-term care. Am J Speech Lang Pathol. 2017 Nov; 26(4): 1213-24.

27. Fujiu-Kurachi M, Fujiwara S, Tamine K, Kondo J, Minagi $\mathrm{Y}$, Maeda $\mathrm{Y}$ et al. Tongue generation during tongue-hold swallows in young healthy adults measured with different tongue positions. Dysphagia. 2014 Feb; 29(1): 17-24. 